\title{
In vivo forced expression of myocardin in ventricular myocardium transiently impairs systolic performance in early neonatal pig heart
}

\author{
MARIO TORRADO ${ }^{1}$, ALBERTO CENTENO ${ }^{2}$, EDUARDO LÓPEZ ${ }^{2}$ and ALEXANDER T. MIKHAILOV ${ }^{1, *}$ \\ ${ }^{1}$ Developmental Biology Group, Institute of Health Sciences, University of La Coruña and \\ ${ }^{2}$ Experimental Surgery Unit, University Hospital "Juan Canalejo", La Coruña, Spain
}

\begin{abstract}
The aim of this study was to determine the effects of forced expression of myocd-A in the left ventricular (LV) myocardium on cardiac performance in early neonatal piglets. LV transfection with the gene for homeodomain only protein (hop), an antagonist of myocdmediated activities, was also performed. Gene delivery was performed in 6-day-old piglets using a low-traumatic, catheter-based, video-assisted procedure developed by us for direct intramyocardial injections of plasmid DNA into 3-4 target areas of the ventral LV free wall (LVFW). Two isoforms of porcine myocd were identified, cloned and characterized: the exon 11-lacking myocd$A$ and its larger exon 11-containig variant, myocd-B. In neonatal piglets, myocd-A seems to be a cardio-predominant isoform enriched in the LVFW/septum, whereas the myocd-B isoform is detected not only in the heart but also in various smooth muscle cell-containing tissues. Intramyocardial myocd-A gene delivery resulted in forced transgene expression in the target areas of the LVFW as compared to controls. On day 2 post-delivery, a marked decrease of LV-end systolic pressure values (an accepted marker for impaired LV function) was observed in myocd-Atransfected piglets as compared to hop-transfected and control groups. In addition, forced myocd$A$ expression in the LVFW caused abnormal ECG. A significant up-regulation of the gene for fetalpredominant muscle light chain $3 \mathrm{~F}$ myosin was detected in myocd-A-transfected LVFWs harvested on day 2 post-delivery. Extended analysis on day 7 post-delivery revealed a drop decrease in myocd-A transgene expression in target LVFW regions which was correlated with normalization of the LV systolic parameters in experimented piglets.
\end{abstract}

KEY WORDS: myocardin, gene delivery, myocardium, transcription factor, cardiac hypertrophy

"The heart is the first organ to form and function in the embryo, and all subsequent events in the life of the organism depend on the heart's ability to match its output with the organism's demands for oxygen and nutrients"

(Olson, 2004).

\section{Introduction}

Myocardin (myocd) is a cardiac and smooth muscle-selective co-activator for the ubiquitous transcriptional factor, serum response factor (SRF). In the course of embryonic development, the onset of myocd expression coincides with specification of early cardiac progenitors in the cardiac crescent. Thereafter, myocdis expressed throughout the heart and in various subsets of smooth muscle cells (SMCs) (Creemers et al., 2006a).
In Xenopus embryos, expression of a dominant-negative mutant of myocd(Wang etal., 2001) or inhibition of myocd activity by morpholino knockdowns (Small et al., 2005) disrupts cardiac development and abolishes cardiac marker gene expression. However, mouse embryos lacking myocd have a normal heart ( $\mathrm{Li}$

\footnotetext{
Abbreviations used in this paper: ANF, atrial natriuretic factor; EDP, enddiastolic pressure; ESP, end-systolic pressure; Hop, homeodomain only protein; LV, left ventricular; LVFW, LV free wall; myocd, myocardin; qRTPCR, quantitative real-time RT-PCR; RV, right ventricular; SMC, smooth muscle cell; SRF, serum response factor; MLC3F, myosin light chain $3 \mathrm{~F}$ isoform; CNN1, smooth muscle calponin 1 basic isoform; TAGLN, transgelin (SM-22); ACTG2, smooth muscle actin gamma 2 isoform; $Q$, poly $(\mathrm{Q})$-rich tract; SAP, SAF-A/B, Acinus and PIAS motif; TAD, transactivation domain; aa, amino acid residues; EtBr, ethidium bromide.
}

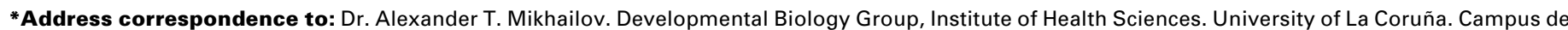
Oza, Building "El Fortín", As Xubias s/n. 15006 La Coruña, Spain. Fax: + 34-981-138-714. e-mail: margot@udc.es
}

Final author-corrected PDF published online: 30th January 2008. 
et al., 2003) probably due to redundancy of myocd-related factors (Wang et al., 2002), which are expressed in the developing mouse, but not in the developing Xenopus heart (Small et al., 2005). Recently, it was found that myocdis transiently expressed within the somites of mouse embryos. However, forced myocd expression in in vitro cultured skeletal myoblasts resulted in repression of activity of the muscle-specific myogenic factors and concurrent activation of SMC-specific genes (Long et al., 2007).

It is widely accepted that myocd is a regulatory factor that plays pivotal roles in both cardiac and SMC gene expression (recently reviewed in: Liu and Olson, 2006; Pipes et al., 2006; Parmacek,


Fig. 1. Procedure of catheter-based video-assisted intramyocardial injections of Indian ink suspension into the left ventricular free wall (LVFW) of 6-day-old piglets. (A) Positions for trans-cutaneous insertion of the endoscopic cannula (EDC) and fiber-optic device (FOD) into left chest cavity. The positions were selected to access and visualize the ventral surface of the heart. (B) Both EDC and FOD are inserted at the positions indicated. (C,D) Intramyocardial injecting controlled by realtime video-signals. $L V$, left ventricle. $(\mathbf{E}, \mathbf{F}, \mathbf{G})$ Ventral, lateral and dorsal view of the heart at the 7th day after Indian ink delivery to the ventral LVFW. Arrows, injection points; RV, right ventricle. $\mathbf{( H , I )}$ Histological sections showing Indian ink infiltration of the myocardium at injection points. Haematoxylin-eosin staining. Bar, $100 \mu \mathrm{m}$.
2007). In Xenopusembryos, ectopic myocdexpression results in activation of cardiac muscle genes throughout the embryo, including spinal cord neurons (Wang et al., 2003), or in animal cap tissue (Small et al., 2005). In cultured mammalian cells, depending on the particular cell type in which myocd expression is induced, different subsets of SRF-dependent SMC and cardiac muscle genes can be activated (Chen et al., 2002b; Du et al., 2003; Wang etal., 2003; Yoshida et al., 2003; Hendrix et al., 2005; Pipes etal., 2005; van Tuyn etal., 2005, 2007a,b; Creemers etal., 2006a,b; Chow et al., 2007).

However, although myocd is required for cardiac and SMC gene expression and differentiation, it is not sufficient to cause other cell types to adopt a cardiac or SMC expression phenotype (Yoshida et al., 2004). In this regard, myocd can initiate the expression of cardiac muscle-specified genes in cultured fibroblasts, but only in the presence of the co-expressed small ubiquitinlike modifier, SUMO-1 (Wang et al., 2007). Undifferentiated liverderived stem cells acquire a cardiomyocyte phenotype when cocultivated in vitro with neonatal cardiomyocytes which, in turn, results in the de novo activation of cardiac transcriptional factors including myocd(Muller-Borer et al., 2007). These and other data (Oh et al., 2004; Callis et al., 2005; Creemers et al., 2006a) strongly suggest that additional factors are required to promote myocardin's role in cardiogenic gene expression.

In addition to its roles in the regulation of cardiac and SMC differentiation and gene expression, myocd has also been implicated in remodeling of the postnatal heart in response to either physiological or pathological stresses during heart ageing, cardiomyopathic progression, and at heart failure (see Table 1 and references therein). Physiological and experimental cardiac hypertrophy is accompanied by marked myocd up-regulation in ventricular myocardium. In this regard, in vitro forced expression of myocd in neonatal ventricular cardiomyocytes increases cell size and expression of molecular markers of cardiac hypertrophy (Badorff et al., 2005; Xing etal., 2006). Glycogen synthase kinase $3 \beta$, suppressor of cardiomyocyte hypertrophy (Michael et al., 2004), phosphorylates MYOCD, thus antagonizing its pro-hypertrophic activity (Badorff et al., 2005).

Taken together, these studies all point to a redeployment of myocdin processes of postnatal cardiac remodeling (Torrado et al., 2003; Torrado et al., 2005a; Oka et al., 2007). While some potential insights into MYOCD/SRF-dependent pathways were described in cultured neonatal cardiomyocytes, the functional contributions of myocdsignaling to physiological cardiac hypertrophy in the neonatal heart in vivo remain unknown.

The primary goal of the present study was to directly assess the possible roles of MYOCD in the neonatal porcine heart in vivo. To this end, a new controlled local delivery of myocd-A-expressed plasmids to left ventricular (LV) myocardium was developed. Using this low traumatic technique, the effects of transfectionmediated myocd- $A$ overexpression in the LV myocardium on cardiac performance and gene expression were monitored in neonatal pig hearts. As additional experimental treatments, LV transfection with the gene for homeodomain only protein (hop), an antagonist of myocd-mediated SRF activities (Kook and Epstein, 2003), was also performed.

Here we show that in vivo forced expression of myocd- $A$ in ventricular myocardium transiently impairs systolic performance in neonatal pig hearts. Given that end-stage heart failure is 
accompanied by an increase in myocdexpression (see Table 1), these results demonstrate, for the first time, that myocardial myocd overexpression might represent a maladaptive response to cardiac stress.

\section{Results}

The use of a newly developed catheter-based device results in efficient intramyocardial delivery in closed-chest neonatal piglets

Direct intramyocardial delivery is attractive because specific myocardial regions can be targeted and high local concentrations of the transgene can be achieved. However, direct myocardial gene delivery often requires aggressive surgical interventions resulting in a low survival rate of experimental animals.

We developed and evaluated the feasibility of a catheterbased video-assisted surgical approach for direct intramyocardial gene transfer in closed-chest 6-day-old neonatal piglets (Fig. 1AD). For catheter optimization studies, needle length $(2-5 \mathrm{~mm})$, injection volume (50-200 $\mu \mathrm{l} / \mathrm{point})$, and the localization/distribution of the delivered trackers (Indian ink suspension or $\beta$-galactosidase ( $\beta$-gal) carrying plasmid DNA) were assessed two and seven days after injections.

The intramyocardial injections were well tolerated in all animals. Sometimes, injections provoked isolated ventricular ectopic beats but no sustained dysrhythmia. In all delivery experiments, piglets became fully ambulatory during the first $30 \mathrm{~min}$ after removal of anesthetics. Histological analysis revealed no evidence of inflammatory cell infiltration or micro-infarctions in LVFW regions injected with naked $\beta$-gal plasmid DNA at the 7th day post-delivery. However, injections of the $\beta$-gal plasmid DNA solution supplemented by in vivo-jetPEI transfection reagent (at $\mathrm{N} / \mathrm{P}=10$ ) did result in signs of inflammation and calcium deposition in the targeted LVFW regions.

The optimal needle length in these neonatal piglets with a LVFW thickness of 7.3 \pm 0,3 mm (Torrado et al., 2004) was determined to be $4 \mathrm{~mm}$ and, using this needle length, the optimal injection volume was determined to be $100 \mu \mathrm{l} /$ point. Under our conditions, 3-4 discrete injections (100-150 $\mu$ leach), spaced $1 \mathrm{~cm}$ apart, in each LVFW resulted in distribution of Indian ink tracker across $\sim 50 \%$ of the ventral LVFW surface (Fig. 1E).

Furthermore, to determine more precise quantitative data regarding transfection efficiency, myocardial delivery of $\beta$-gal plasmid DNA was performed using the same injection protocol. Protein extracted from injected and adjacent regions of each LVFW was assayed for $\beta$-gal enzymatic activity. The same or an even more vast distribution of $\beta$-gal enzymatic activity was found in the corresponding LVFWs as compared to that in myocardium injected with Indian ink suspension. These results were further confirmed by reverse transcription-polymerase chain reaction (RT-PCR) detection of $\beta$-gal expression in the injected LVFW regions (data not shown).

In conclusion, the use of the optimized intramyocardial delivery device with video-assisted guidance resulted in a relatively efficient delivery of the trackers used to target the regions of the LVFW with $100 \%$ recovery of experimental piglets.

\section{Which porcine myocardin gene to use for delivery?}

Previously, we have provided evidence for the existence of two myocd transcript variants in four cardiac cambers, as well as in aorta, pulmonary vein, and lung of neonatal piglets (Torrado etal., 2003). In this work, two porcine myocd variants were cloned, sequenced and designated, in accordance with our tentative classification (Torrado et al., 2003), as a myocd- $A$ and its spliced variant $m y o c d-B$, containing a 144-bp insertion due to the presence of an alternative exon (marked 11 in Fig. 2A and Fig. 4A).

Using RT-PCR, both myocd- $A$ and $m y o c d-B$ transcripts were detected in all heart chambers of 6-8-day-old neonatal piglets with relative myocd $A /$ myocd- $B$ expression ratio (fold expression) of $1: 9 \pm 2.8$ (Fig. 2B, C). Myocd- $B$ was also found to be expressed in aorta, pulmonary veins, lung and spleen, but its expression was not detectable in skeletal muscle, liver and kidney. In contrast, in addition to being expressed in myocardium, myocd- $A$ mRNA, was weakly detected only in aorta (Fig. 2C).

To confirm that each of the two myocd transcripts can be efficiently translated into protein, the corresponding myc/Histagged cDNAs were expressed in vitroand the encoded polypeptides were detected by SDS-PAGE followed by Western blot with our lab-derived anti-CT-MYOCD-A (named AB531; see Fig. 4B, C) or anti-myc antibodies. On blots, both MYOCD-A and MYOCD$B$ proteins were detected by each of the antibodies used (Fig. 2D, $\mathrm{E})$. The experimentally determined molecular weight (MW) value of myc/His-tagged MYOCD-A (128 kDa) and MYOCD-B (133 $\mathrm{kDa}$ ) is an $\sim 28 \mathrm{kDa}$ higher than the predicted sequence-derived

TABLE 1

\section{MYOCARDIN EXPRESSION LEVELS AT HEART AGING, VENTRICULAR HYPERTROPHY AND HEART FAILURE}

\begin{tabular}{|c|c|c|c|c|}
\hline Model & Cardiac phenotype & Phase/Clinical syndrome & Myocardin (up/down) & Reference \\
\hline Young vs newborn piglets & VH due to early postnatal growth & Physiological VH & transcript (up) & Torrado et al., 2003 \\
\hline Old $v s$ young adult mice & Aging heart & NA & transcript (up) & Zhang et al., 2004 \\
\hline Old vs young adult humans & Aging heart & NA & transcript (up) & Zhang et al., 2004 \\
\hline Adult TAB mice & VH due to aortic stenosis & No signs of HF & transcript (up) & Xing et al., 2006 \\
\hline Calcineirin-transgenic mice & VH due to calcineurin overexpression & Susceptible to sudden death & protein (up) & Xing et al., 2006 \\
\hline \multirow[t]{2}{*}{ SRF-deficient adult mice } & DCM due to disruption of the SRF gene & Early-stage DCM & transcript (down) & Parlakian et al., 2005 \\
\hline & & End-stage HF & transcript (up) & \\
\hline Dox-injected piglets & DCM due to Dox cardiotoxic effects & End-stage $\mathrm{HF}$ & transcript (up) & Torrado et al., 2003 \\
\hline F vs NF human hearts & IDCM & End-stage HF & transcript (up) & Torrado et al., 2003 \\
\hline \multirow[t]{2}{*}{ F vs NF human hearts } & IDCM & No signs of HF & protein (no change) & Xing et al., 2006 \\
\hline & IDCM & End-stage $\mathrm{HF}$ & protein (up) & \\
\hline
\end{tabular}

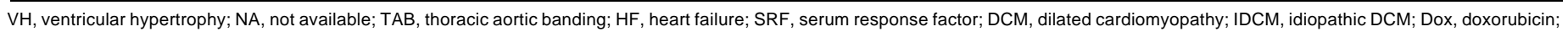
$F$, failing; NF, non-failing heart. 
MW of porcine MYOCD-A ( 101 kDa) and MYOCD-B ( 105 kDa), respectively.

To the best of our knowledge differences in apparent versus deduced MW of MYOCD proteins have not been recognized in previous studies on mouse myocd (Creemers et al., 2006b; Xing et al., 2006; Wang et al., 2004, 2007). On the other hand, one can deduce from published SDS-gel images (see Qiu et al., 2005; Li et al., 2007) that an apparent MW of FLAG-tagged mouse MYOCD seems to be between 120-130 kDa that is similar to the MW value of myc/His-tagged porcine MYOCD-A (this work). It seems that the presence of $\mathrm{myc} / \mathrm{His}$ residues (see Fig. 2D,E) or FLAG epitopes (see Fig. 3B) in expressed MYOCD proteins contributes only to a minor extent to analomous slow migration of the pig MYOCD proteins in SDS-PAGE gels. Of note, the experimentally estimated 5-kDa difference between the myc/His-tagged 128-kDa MYOCD-A and the 133-kDa MYOCD-B closely agreed with the theoretical 4-kDa size difference between these two isoforms of porcine MYOCD (i.e., $101 \mathrm{kDa} v s 105 \mathrm{kDa}$ ).

Furthermore, using AB531-antibodies, a single 145-kDa band was immuno-decorated on blots of electrophoresed SDS-lysates

A
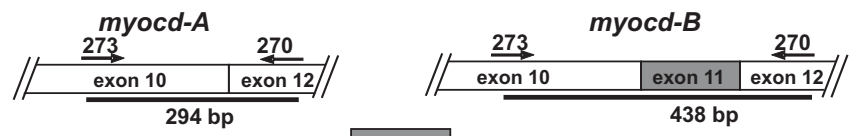

exon 11

2044 ACCTCAGGGGCACACGAGGGCCATTCTCCAAGCTTCTCCAACCAGCCCTCCAGCCTCCACCCCCССTTCTCT 2115 $\begin{array}{llllllllllllllllllllllllll}682 & \text { T } & \text { S } & G & \text { A } & \text { H } & \text { E } & \text { G } & \text { H } & \text { S } & \text { P } & \text { S } & \text { F } & \text { S } & \text { N } & \text { Q } & \text { P } & \text { S } & \text { S } & \text { L } & \text { H } & \text { P } & \text { P } & \text { F } & \text { S } & 705\end{array}$ 2116 GGAGCCCAGGCAGACAGCAGTCAAGGTGCCGGGGGCAACCCTTGTCCTAAAAGCCCAGGTGTGCAGCAAAAG 2187 $\begin{array}{llllllllllllllllllllllllllll}706 & \text { G } & \text { A } & \text { Q } & \text { A } & \text { D } & \text { S } & \text { S } & \text { Q } & \text { G } & \text { A } & \text { G } & \text { G } & \text { N } & \text { P } & \text { C } & \text { P } & \text { K } & \text { S } & \text { P } & \text { G } & \text { V } & \text { Q } & \text { Q } & \text { K } & 729\end{array}$

B

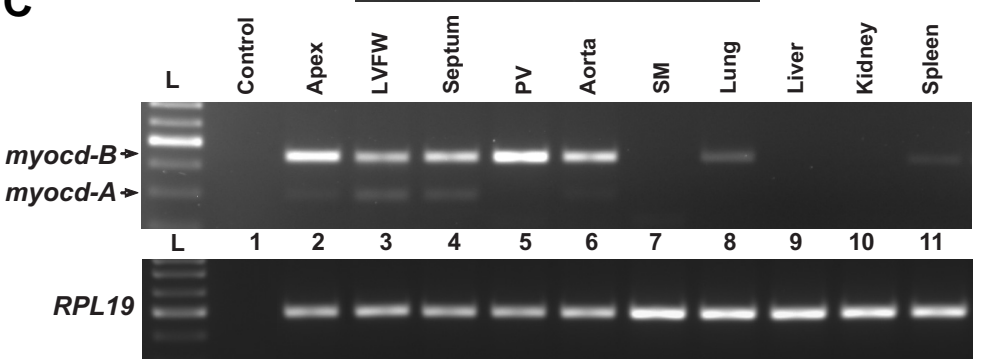

D

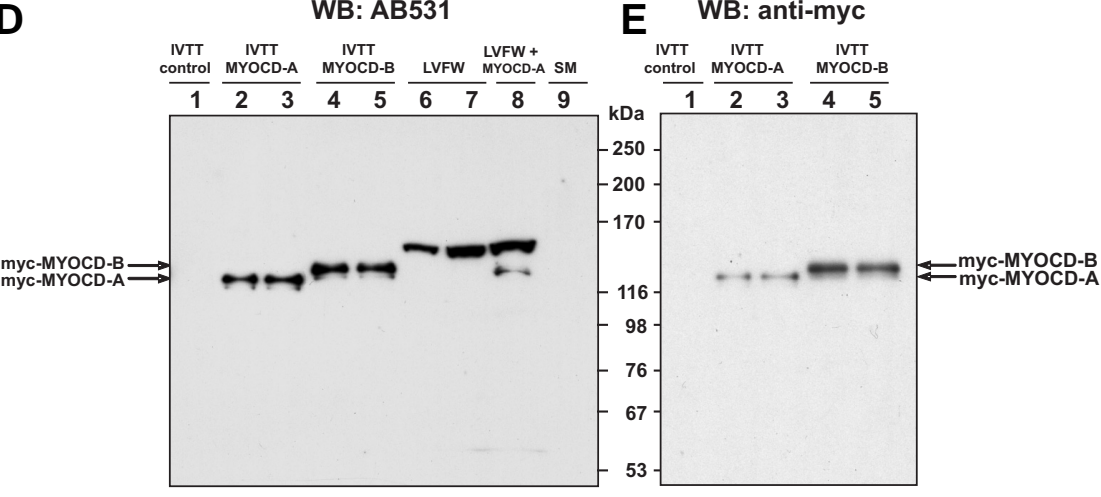

from LV but not from skeletal muscle of neonatal piglets (Fig. 2D). The specificity of this cross-reactivity was confirmed by preabsorption of AB531 with the competing peptide, which blocked immuno-detection of the heart-derived 145-kDa band. Of note, the fragment 5 of pig myocd-A used as immunogen to generate AB531 antibodies (see Fig. 4B) is identical for both porcine myocd- $A$ and $m y o c d-B$ isoforms. Therefore, it was not surprising that these antibodies recognized both in vitro synthesized MYOCDA and MYOCD-B proteins (see Fig. 2D). However, we observed the only one $145-\mathrm{kDa}$ band on piglet LV-derived immunoblots (see Fig. 2D). Although unproved as yet, we suggest that the band with an apparent MW of 145-kDa might correspond to an endogenous MYOCD-B protein. In this respect, we found that the myocd- $B$ mRNA is much more abundant than the myocd- $A$ transcript in the LV (see Fig. $2 \mathrm{~B}, \mathrm{C}$ ) suggesting that under our experimental conditions presumably MYOCD-B protein might be detected on LV-derived blots. Differences in an apparent MW between in vitro expressed MYOCD-B (MW 133-kDa) and heartderived protein (MW $145 \mathrm{kDa}$ ) may be due to post-translational modifications of the latter, such as symolation (Wang et al., 2007), or due to its conformation changes. In this context, SUMO-modified transcription factors have a $\sim 20$ kDa higher apparent MW as compared to unmodified proteins (Eloranta and Hurst, 2002; Sapetschnig et al., 2002).

Collectively, the results revealed a differential expression of myocd- $B$ versus myocd- $A$ genes in the piglet $L V$ myocardium and a modest but steady level of $m y o c d-A$. In neonatal piglets, the exon 11 . lacking $m y o c d-A$ seems to be a cardiac-predomi-

Fig. 2. Analysis of myocd-A and myocd-B expression in vivo and in vitro. (A) Positions of the oligonucleotides 273 and 270 (horizontal arrows) over porcine myocd-A and myocd-B exon 10 and exon 12 (white boxes) that have been used in RT-PCR analysis. Spliced exon 11 in myocd-B is boxed in grey. Bold-faced black lines indicate 294-bp and 438-bp PCR fragment of the pig myocd-A and myocd-B, respectively (top part). Nucleotide and deduced amino acid sequences of the myocdB exon 11 (lower part). (B) Representative semiquantitative RT-PCR for myocd-A, myocd-B and RPL19 genes in left and right ventricles ( $L V, R V)$ and atria ( $L A, R A)$ of 6-day-old piglets. (C) Expression of myocd-A and myocd-B in $L V$-derived tissues and various noncardiac tissues/organs of 8-day-old piglets as determined by semiquantitative RT-PCR. PV, pulmonary vein; SM, skeletal muscle; L, DNA ladder; Control, non-template PCR. (D,E) Western blot analysis of in vitro (IVTT) expressed myc-tagged myocd-A (lane 2, 3) and myocd-B (lane 4, 5) cDNAs using anti-CT-MYOCD (AB531) and anti-myc antibodies, respectively. Lane 1 (IVTT control), non-plasmid DNA negative control. In addition, extracts prepared from LVFW of 8-day-old neonatal piglets were probed with anti-CT-MYOCD antibodies (right part of D): lane 6, LVFW extract (15 $\mu \mathrm{g}$ of total protein); lane 7, LVFW extract (30 $\mu \mathrm{g}$ of total protein); lane 8, IVTTexpressed myocd-A protein was loaded together with LVFW extract (LVFW+MYOCD-A); lane 9, extract from skeletal muscle (SM, $30 \mu \mathrm{g}$ of total protein) of 8-day-old neonatal piglets. 
nant isoform enriched in LVFW and septum whereas the exon 11-containing myocd- $B$ isoform is expressed not only in the heart but also in SMCcontaining tissues such as aorta, pulmonary veins, lung and spleen (see Fig. 2 A-C). Of note, forced expression of similar (see Fig. 4A) human myocd- $A$ and $m y o c d-B$ isoforms in myocardial scar fibroblasts didn't reveal significant differences in their transactivating capacity, making the biological relevance of myocd-B exon 11 uncertain (Tuyn et al., 2007b). Additionally, we reasoned that since the baseline levels of $m y o c d-A$ expression are low in the piglet myocardium, the consequences of forced myocd- $A$ expression in the LVFW could be more palpable than in case of myocd- $B$ delivery. Given also that comparison of the effects and quantitative measurements of the delivered myocdvariants could significantly interfere with endogenous myocd transcripts/proteins, for gene delivery experiments we used the myocd-A-carrying plasmid.

\section{Intramyocardial delivery of myocardin-A results in transiently impaired systolic performance}

Using the optimal delivery protocol developed in the first part of the work, naked plasmids encoding myocd- $A$ gene, hop gene or vector itself were injected directly into target sites of the LVFW of 6-dayold neonatal piglets. Two days after delivery, nine regions of each injected LVFW (Fig. 3A) were assayed individually by Western blot with anti-FLAGantibody (Fig. 3B) and RT-PCR (Fig. 3C,D). To ensure the specificity of RT-PCR for delivered CDNAs, we used RT-PCR with discriminating primer sets. In all RT-PCR experiments, we observed highly specific amplification of only the template specific for the discriminating downstream primer complementary to the FLAG-tagged myocd-A (Fig. 3C, D) or hop sequence (data will be described elsewhere).

All myocd- $A$-transfected LVFWs $(n=10)$ harvested on day 2 post-delivery demonstrated transgene expression detectable by RT-PCR. The relatively high ectopic expression of the FLAG-myocd- $A$ was restricted almost exclusively to segments 3,5 , and 6 that included the injection sites. FLAG- myocd-Atransgene expression was not found in other tissue segments except in the two LWFWs, which also revealed transgene expression in region 2 and 4 (Fig. 3C). The results of non-RT control PCR runs demonstrated that the FLAG- $m y o c d-A$ PCR product was amplified from LV cDNA, but not from plasmid DNA (see Fig. 3D). On average, the estimated extent of FLAGmyocd- $A$ expression was $40 \%$ of the overall area of the ventral LVFW. Target delivery regions of LVFWs (i.e., region 3, 5, and 6) injected with hop or empty vector were consistently negative for FLAG- $m$ yocd- $A$ transgene expression (data not shown).

The myocd-A/myocd- $B$ mRNA ratio in FLAGmyocd- $A$-delivered LVFW regions was significantly affected as compared to that in myocardium of PBS-
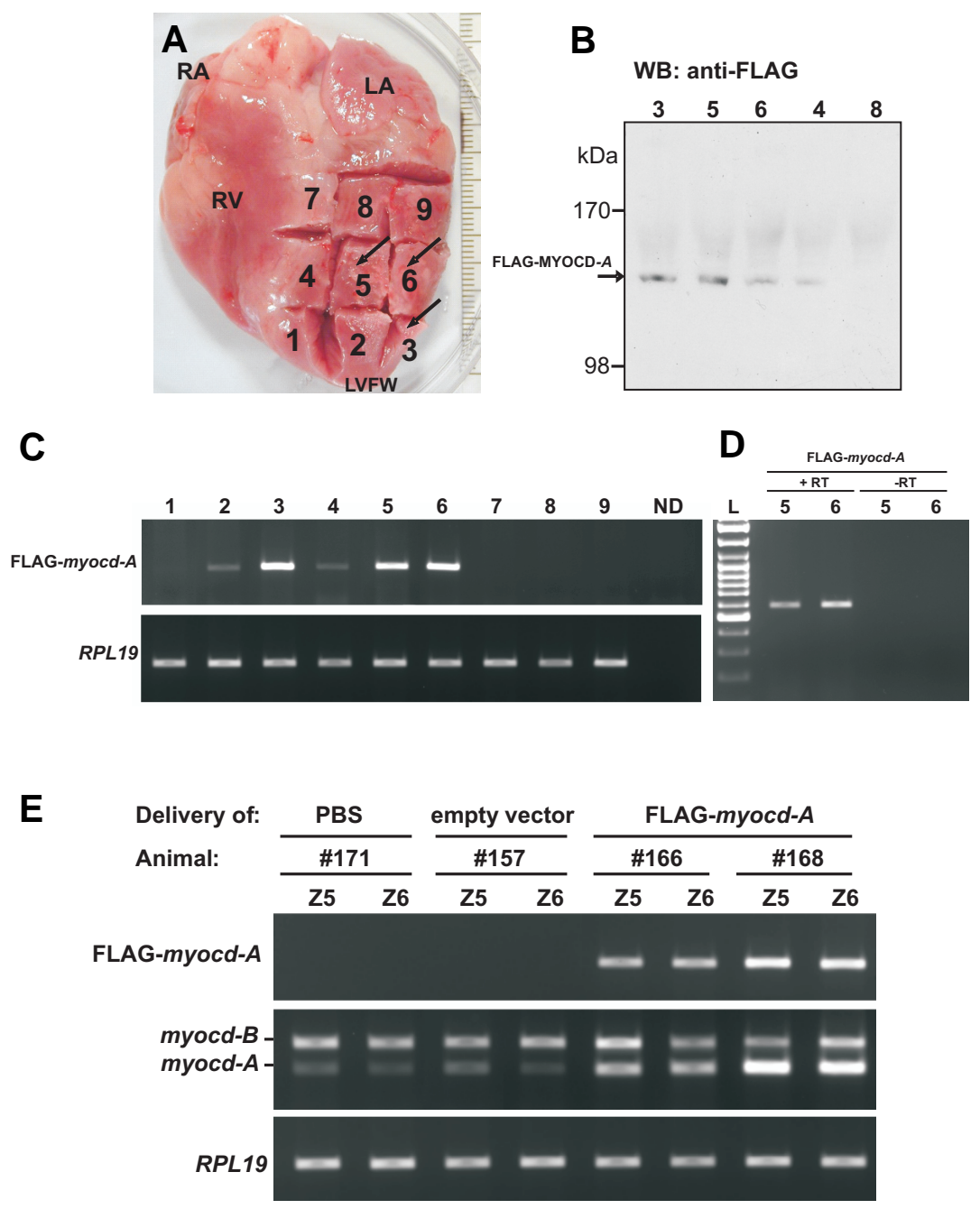

Fig. 3. Representative analysis of FLAG-tagged myocd-A expression in the left ventricular free wall (LVFW) myocardium $48 \mathrm{~h}$ after gene delivery (8-day-old piglets). (A) myocd-A transfected LVFW (arrows, injection positions) was dissected into nine fragments (1-9). $R V$, right ventricle; $R A$ and $L A$, right and left atrium, respectively. (B) Western blot detection of FLAG-tagged MYOCD-A protein in dissected target $(3,5,6)$ and adjacent $(4,8)$ regions of myocd-A transfected $L V F W$. (C) Semiquantitative RT-PCR analysis of FLAG-tagged myocd-A transcript levels in nine LVFW segments (after normalization of the CDNA templates to RPL19 expression). ND, non-delivered myocardium. (D) Control of plasmid DNA contamination in RNA preparations used in downstream $R T$ T-PCR assays (see C,E). Total $R N A$ was isolated from the target regions 5 and 6 of myocd-A transfected LVFW (see A). Four $\mu g$ of each RNA preparation were reverse transcribed $(R T)$ in $20 \mu$ reaction mix. One $\mu$ l of each $R T$ reaction $(+R T)$ and $1 \mu \mathrm{g}$ of each $R N A(-R T)$ were subsequently used in a standard $P C R$ reaction for the amplification of the FLAG-tagged myocd-A message. Equal volumes of each $P C R$ reaction were assessed on a $2 \%$ agarose gel and stained with EtBr. $L$, GeneRuler DNA ladder mix (Fermentas). (E) Semiquantitative RT-PCR analysis of total RNAs isolated from the indicated zones $(Z 5, Z 6)$ of LVFWs injected with PBS (piglet \#171), empty vector (piglet \#157) or FLAG-tagged myocd-A (piglet \#166 and \#168) plasmids. Upper part, recombinant FLAG-myocd-A transcript levels. Middle part, endogenous/recombinant myocd-A and endogeneous myocd-B transcript levels. Lower part, RPL19 transcript levels. 
A

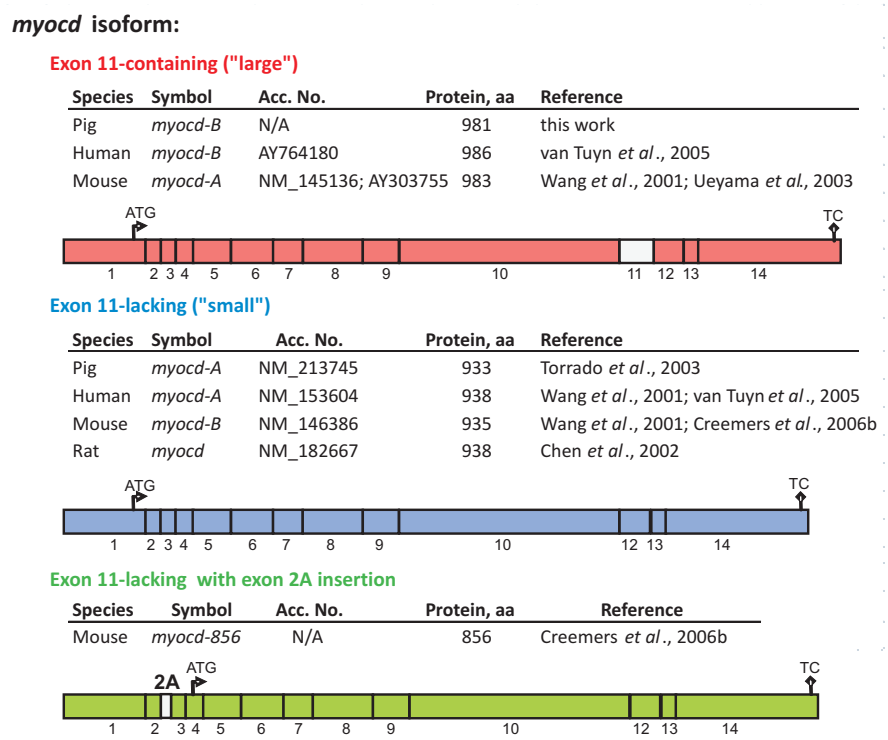

B

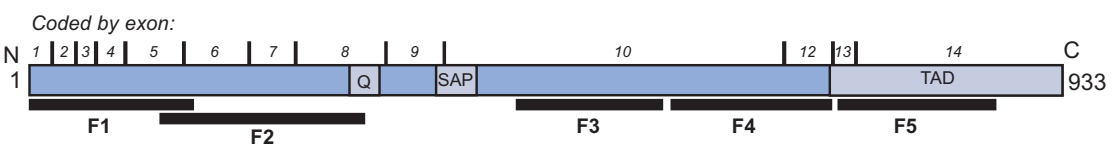

C

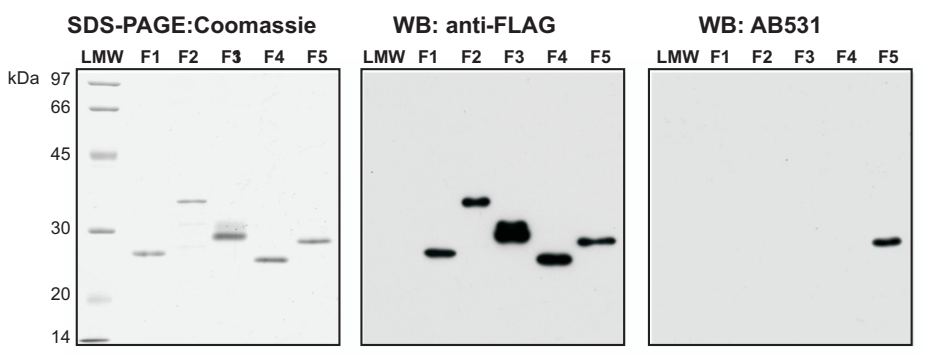

Fig. 4. Alternative splicing yields different myocd transcripts with similar C-termini coded by exons $\mathbf{1 3}$ and 14. (A) Schematic diagrams of three different myocd isoforms (marked respectively in red, blue and green) detected in cardiac and SMC-containing tissues. Myocd, gene name approved by HUGO Gene Nomenclature Committee. Transcript symbols are indicated in accordance with original definitions. 1-14, exons (alternative exon $2 \mathrm{~A}$ and 11 are boxed in white). In each myocd isoform, the transactivation domain is coded by similar exons 13 and 14. The exon 11-lacking myocd A (blue) and exon 11containing myocd-B (red) have similar N-terminal peptide sequences. Green, Nterminus truncated myocd isoform. ATG and TC, translation initiation and termination codon, respectively. aa, amino acid residues. N/A, not available. (B) Five fragments (F1-5 marked by bold-faced black lines) covering the major part of the sequence of porcine MYOCD-A were cloned, expressed and purified as described in Materials and Methods. The C-terminal (CT) fragment 5 of porcine MYOCD-A (aa 731-873) was used, as immunogen, to generate $A B 531$ antibodies. $Q$, poly(Q)-rich tract. SAP, $\underline{S} A F-A / B$, Acinus and PIAS motif. TAD, transactivation domain. (C) Purified fragment products (F1-5) were separated by SDS-PAGE followed by Coomassie staining of gels (left) or Western blot (WB) with anti-FLAG (center) and AB531 antibodies (right). Anti-FLAG antibodies detect all fragments due to presence of the FLAG epitopes in each expressed protein. AB531 antibodies recognize only the CT fragment 5 used for immunization; no cross-reactively was observed with other MYOCD-Aderived fragments. LMW, Amersham low molecular weight calibration kit. and vector-delivered (Fig. 3E) or age-matched (Fig. 2B,C) piglets. In the target regions, delivered FLAG- $m y o c d-A$ caused an increase of the myocd- $A$ transcript content in direct proportion to the transgene expression level. The amount of the endogenous myocd-BRNA did not change appreciably in $m y o c d-A$-delivered regions (Fig. $3 \mathrm{E})$. Of note, intramyocardial delivery of the vector itself did not influence endogenous myocd$A /$ myocd- $B$ mRNA levels in the target regions of LVFWs. Histological examination did not reveal inflammation or fibrosis in the LVFW target regions from myocd- $A$-delivered piglets (data not shown).

Ventricular systolic function was clearly altered in myocd-A-delivered piglets as compared with that in animals intramyocardially injected with hop-plasmid or the vector itself. Particularly, intramyocardial injections of myocd $A$ resulted in a marked decrease of LVESP, an accepted marker for impaired LV function. In addition, forced myocd-A expression in the LVFW caused abnormal ECG including T-wave inversion (Table 2). In hop-delivered and control groups, there was no change in ECG parameters from gene delivery to final studies, nor were there differences in LVESP values between these groups.

In all animals, LVEDP (a marker for LV compliance) values were the same regardless of whether $m y o c d-A$ or other plasmids were delivered into myocardium. In myocd- $A$ delivered hearts, there was no difference in the heart mass as assessed by the ratios of heart/body weight compared with control piglets (Table 2).

To examine the duration of expression from the delivered myocd- $A$ naked DNA, we have analyzed by RT-PCR the level of FLAG- myocd$A$ mRNA in hearts $(\mathrm{n}=5)$ harvested after 7 days after gene delivery. On day 7 , the transgene expression level in the LV target regions was, on average, 3-fold less than that on day 2 . The systolic parameters were not altered in this animal group as compared to age-matched controls (i.e., 13-day-old piglets; $n=4$ ) transfected with empty vector (data not shown). These experiments show the transient character of $m y o c d-A$ transgene expression in the heart and strongly suggest that only a highly forced myocd-A expression might have a functional consequences on LV systolic performance.

Considering the well-established involvement of myocd in regulating both cardiac and SMC gene expression, we studied in myocd-A-delivered hearts the endogenous expression of a set of the genes previously shown to be bona fide myocd targets. Expression of selected cardiac and SMC marker genes was assessed by qRT-PCR in FLAGmyocd- $A$ - $(n=5)$ versus vector-delivered $(n=4)$ piglets two days after transfection (Table 3; Fig. 5). These gene expression studies were performed using myocardial samples derived from two target region 5 and 6 (see Fig. $3 \mathrm{~A}$ ) of each LVFW transfected with $m y o c d-A$ ( 5 animals) or empty vector 
A

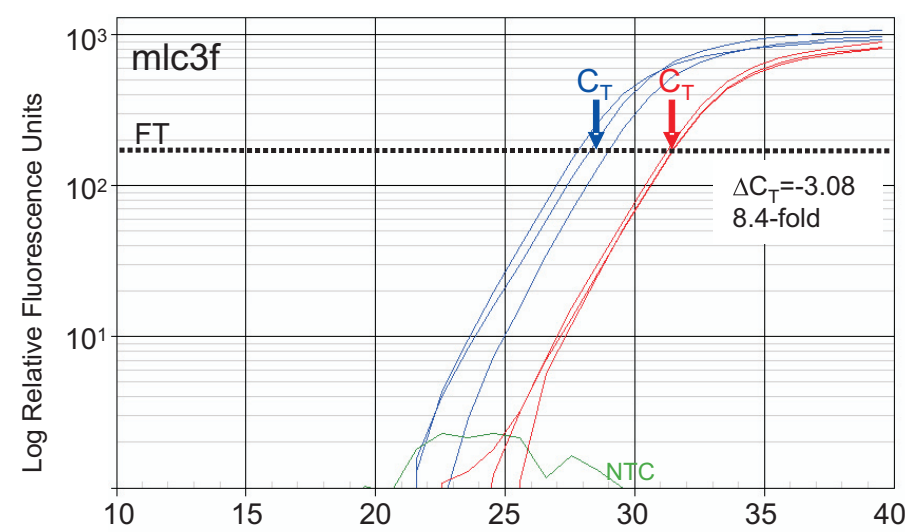

B

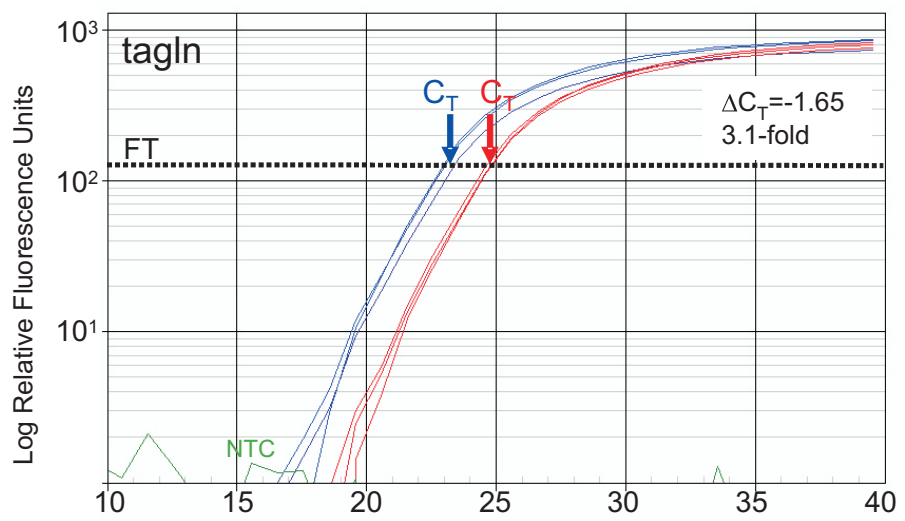

C

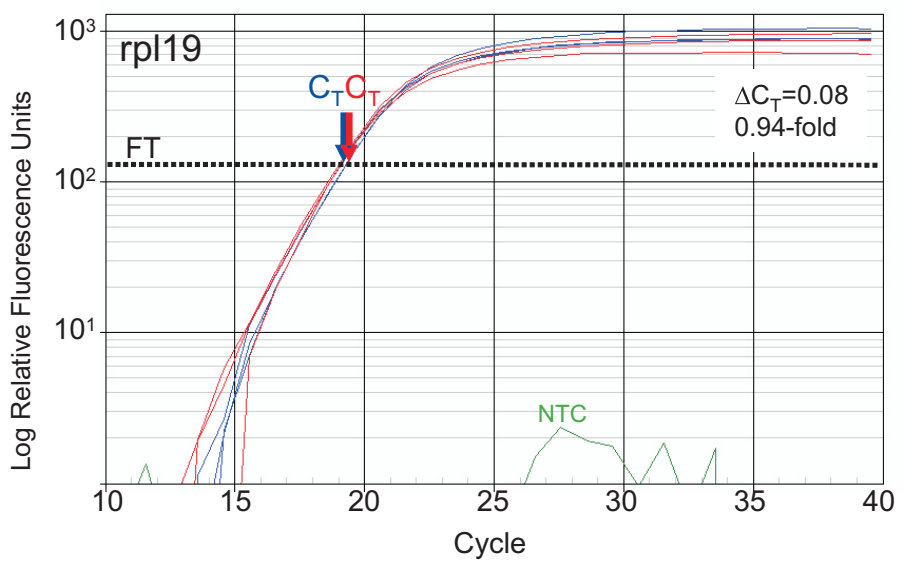

Fig. 5. Representative qRT-PCR analysis of $m / c 3 f(A)$ and tagln (B) expression levels in myocd-A versus vector-transfected left ventricular (LV) myocardium two days after delivery. For each gene, $m R N A$ levels (normalized to (C) the internal standard rpl19) were measured in six samples, each derived from the target region 5 (see Fig. 3A) of three myocd-A- (blue plots) and three empty vector-injected (red plots) LVFWs. $C_{T}$, cycle threshold; $\Delta C_{T}$, differences in threshold cycles for target and reference. Calculated fold-change in mlc3f and tagln expression levels is shown. FT, fluorescence threshold. NTC (green), non template control.
(4 animals). In total, 10 FLAG- $m y o c d-A$ - and 8 vector-delivered samples were processed. The gene for myosin light chain $3 \mathrm{~F}$ ( $m / c 3 x$ ) displayed the most remarkable, over 8-fold up-regulation in response to intramyocardial $m y o c d-A$ delivery (Fig. $5 \mathrm{~A}$; Table 3). Of note, we didn't detect expression of the spliced $\mathrm{m} / \mathrm{c} 1 \mathrm{f}$ isoform in the neonatal myocardium of 8-day-old control and myocd- $A$ transfected piglets. Among the studied SMC marker genes, only trangelin/SM-22 (tag/n) was markedly and statistically significantly stimulated in response to FLAG- $m y o c d-A$ (Fig. $5 \mathrm{~B}$, Table 3 ). The expression levels of the gene for atrial natriuretic factor (ant), smooth muscle calponin 1 (cnn 1 ) and smooth muscle actin gamma 2 (actg2) were also up-regulated in the myocd-A-delivered LVFW regions as compared to those injected with the vector itself, but the differences were not statistically significant.

Collectively, the results indicate that under our experimental conditions myocd-A-delivered hearts shown signs of transiently impaired systolic function that is associated with marked activation of $m c / 3 f$ and taglnexpression in target LVFW regions on day 2 post-delivery.

\section{Discussion}

The recent discovery of MYOCD that modulates the activities of SRF in cardiac and SMC tissues (Wang et al., 2001) has led to intensive investigation into the role that MYOCD can play in heart development and disease and has revealed new combinatory pathways by which MYOCD can regulate cardiac gene expres-

\section{TABLE 2}

CARDIAC PARAMETERS OF NEONATAL PIGLETS INTRAMYOCARDIALLY INJECTED WITH MYOCD-A, HOP, $\beta$-GAL, AND PLACEBO PLASMIDS ON DAY 2 POST-DELIVERY

\begin{tabular}{lcccc} 
Parameter & myocd-A & hop & $\beta$-gal & Vector itself \\
\hline Number of animals & 10 & 6 & 6 & 4 \\
Heart to body weight ratio, x1000 & $6.5 \pm 0.3$ & $6.9 \pm 0.3$ & $7.4 \pm 0.4$ & $6.5 \pm 0.2$ \\
Heart rate (beats/min) & $163 \pm 35$ & $161 \pm 40$ & $174 \pm 31$ & $140 \pm 16$ \\
LV end-systolic pressure (mm Hg) & $37.2 \pm 3.2^{*}$ & $62.2 \pm 4.2$ & $61.8 \pm 3.1$ & $61.0 \pm 3.3$ \\
LV end-diastolic pressure (mm Hg) & $4.6 \pm 1.4$ & $5.6 \pm 1.4$ & $4.3 \pm 1.3$ & $5.5 \pm 1.2$ \\
T-wave ischemic changes, \% & 20 & 0 & 0 & 0
\end{tabular}

${ }^{*} p<0.05$

TABLE 3

\section{RESPONSES OF CARDIAC AND SMC MARKER GENES TO FORCED MYOCD-A EXPRESSION IN LVFW TARGET REGIONS ON DAY 2 POST-DELIVERY}

\begin{tabular}{llcl} 
Gene & Acc. No. & $\begin{array}{c}\text { Fold change } \\
\text { mean } \pm \text { SEM }\end{array}$ & myocd target / reference \\
\hline mlc3f & DQ629159 & $8.1 \pm 1.5^{*}$ & cardiac / Creemers et al., 2006b \\
anf & NM_214260 & $1.2 \pm 0.2$ & cardiac / Xing et al., 2006 \\
cnn1 & NM_213878 & $1.3 \pm 0.2$ & SMC / Chen et al., 2002b \\
tagln & AK234135 & $2.9 \pm 0.3 *$ & SMC / Yoshida et al., 2003 \\
actg2 & AK240464 & $1.4 \pm 0.2$ & SMC / Pipes et al., 2005 \\
\hline
\end{tabular}

qRT-PCR analysis of mRNA levels of target genes (normalized to the internal standard $r p / 19$ in FLAG- yyocd- $A$ delivered LVFW regions (sample size $=10$, see text). Fold change as compared to LVFW regions injected with empty vector. References - bibliographic information used for our selection of the genes as the direct myocd targets. m/c3f, myosin light chain $3 \mathrm{f}$ isoform; anf, atria natriuretic factor gene; $c n n 1$, smooth muscle calponin 1 basic isoform; tag/n, transgelin (SM-22); actg2, smooth muscle actin gamma 2 isoform. * $p<0.05$ 
sion (reviewed in: Liu and Olson, 2006; Pipes et al., 2006; Parmacek, 2007). However, consequences of MYOCD up-regulation during physiological and pathological LV remodeling in the postnatal heart in vivo (see Table 1) have not yet been highlighted. In this study, we present the results of the first step of our approach to determining whether MYOCD up-regulation in the LV of neonatal piglets might have functionally significant consequences.

As previously reported, mouse (Wang et al., 2003), human (Du et al., 2003; van Tuyn et al., 2005) and pig (Torrado et al., 2003) myocdgene can undergo alternative splicing in cardiac and SMCcontaining tissues. Our in silico analysis (Fig. 4A) of the myocd gene transcript variants identified in human, pig, rat and mouse cardiac and SMC-containing tissues shows that: (1) differential splicing does give rise to, at least, three different myocd transcripts: myocd- $A$, myocd- $B$, and $\mathrm{N}$-terminus truncated isoform, (2) both myocd- $A$ (i.e., the exon 11-lacking isoform) and myocd$B$ (i.e., the exon 11-containing isoform) initiate translation at the ATG codon located in exon 1 producing a MYOCD-A "small" (933938 aa) and MYOCD-B "large" (981-986 aa) protein, respectively, and (3) alternative splicing of exon 2 to exon $2 a$, introduces a stop codon in exon 1 so that translation is initiated from the additional ATG codon located in exon 4 generating the 856 aa protein variant. Excepting this $\mathrm{N}$-terminus truncated isoform, all myocd$A / B$ alternative variants contain the domains required for interactions with SRF and myocyte-specific enhancer factor 2 (MEF2), as well as, the transactivation domain. The $\mathrm{N}$-terminus truncated isoform lacks the sequence required for interaction with MEF2 (Creemers et al., 2006b).

In this work, two forms of the porcine myocd were identified, cloned and characterized (see Fig. 2 and Fig. 4A): myocd- $A$ and its larger splice variant, myocd $B$. Both isoforms of porcine $m y o c d$ are expressed in cardiac chambers of the neonatal pig heart with much lower levels of the myocd-Atranscript as compared to those of the myocd- $B$. These results are consistent with the data on expression patterns of the similar myocd- $A$ and myocd- $B$ transcripts in human heart (van Tuyn et al., 2005) but differ from those in mouse. In mouse, the myocd- $B$ isoform (encoded a 935-aa protein) was detected by RT-PCR predominantly in the heart, whereas the N-terminus truncated myocd isoform (encoded a 856-aa protein), in aorta (Creemers et al., 2006b). In this light, in neonatal piglets the myocd- $A$ "small" isoform enriched in LV and ventricular septum seems to be a cardiac-predominant isoform, whereas the myocd- $B$ "large" variant is expressed at comparable levels not only in the heart but also in aorta and pulmonary veins (see Fig. 2 B,C).

Comparison of the human isoforms of myocd (that are similar to porcine myocd- $A$ and myocd- $B$, see Fig. 4A) failed to detect differences in their transactivating capacity (van Tuyn etal., 2005, 2007b). The present study demonstrates that one-time direct intra-myocardial administration of naked plasmid encoding the porcine myocd $A$ results in the sustained expression of the delivered gene in the target regions of the piglet LVFW on day 2 post- delivery. In turn, this forced myocd-A expression in the LV myocardium results in impaired systolic function. Notably, LVFW delivery of the hop gene that can diminish the cooperativity between MYOCD and SRF (Chen et al., 2002a; Shin et al., 2002) or empty vector did not result in altered ventricular performance. FLAG-tagged myocd- $A$ expression was significantly downregu- lated in target $L V$ regions 7 days after delivery that coincided with full recovery of $L V$ systolic performance in experimented piglets. In fact, we demonstrated that the injection of naked myocd- $A$ DNA, whose high transgene expression could be detected within first several days post-delivery, was enough to trigger a transient LV systolic dysfunction. How can this effect be explained? Impaired LV performance might have been most easily rationalized by forced expression of the myocd- $A$ isoform, the level of which is much lower in normal piglet (this work) and human (van Tuyn et al., 2005) LV myocardium in comparison to levels of the spliced myocd- $B$ isoform. Thus, one possibility is that selectively altered expression of the myocd-A over baseline may be non-physiological for rapid postnatal remodeling of the LV myocardium. In this sense, we detected a significant $m c / 3 f$ up-regulation in neonatal porcine myocardium in response to $m y o c d$ - $A$ delivery (see Table 3, Fig. 5A). Although mc/3f expression was previously thought to be restricted to fast skeletal muscles, later the gene was found to be also expressed in developing mouse heart. Its expression is down-regulated after birth and no traces of the MLC3F protein was detected in adult mouse heart (Kelly et al., 1995; 1998; McGrew et al., 1996). It is formally possible that overexpression of the fetal-predominant MLC3F fast myosin isoform in neonatal LV-myocardium in response to $m y o c d-A$ delivery might interfere with other MLC proteins altering, in turn, the contractile performance of growing piglet myocardium. We cannot also discount the possibility that in porcine neonatal myocardium the myocd- $A$ / myocd-Bratio might act as a "dimmer switch" to fine-tune expression levels of a set of SRF-dependent genes. In this sense, the shifting of the myocd-A/myocd-Bratio in myocd-A-delivered LVFW regions in favor of $m y o c d-A$ (see Fig. $3 \mathrm{D}$ ) may have maladaptive consequences for LV functioning.

An additional influence that might act to impair systolic performance is that under our experimental conditions the target tissue to be transfected by the myocd- $A$ DNA was not only proper LV myocardium but also its outer-most layer including the coronary arteriolar network. In this context, MYOCD overexpression in human vascular SMCs induced a hypercontractile phenotype by enhancing the expression of SRF/MYOCD-dependent contractile genes (Chow et al., 2007). In this work, we detected the upregulation of several SMC-specific contractile filament genes (especially, tag/n/SM22) in response to myocd-A delivery (see Table 3, Fig. 5B). Therefore, it is not unreasonable to speculate that a possible "contaminant" myocd-A overexpression in both superficial and intramyocardial arteriolar network could promote a hypercontractile SMC phenotype in LV microvasculature, which, in turn, might result in disturbance of LV myocardium perfusion and contractile performance. At present, we have no direct experimental results supporting this hypothesis, and only further work will clarify whether such mechanism is really operating.

Forced myocd expression in cultured neonatal ventricular cardiomyocytes resulted in cell hypertrophy and induction of endogenous anf transcription (Badorff et al., 2005; Xing et al., 2006). In this work, neither histological signs of ectopic cardiomyocyte hypertrophy nor appreciable anfgene up-regulation were observed in target LVFW regions two days after myocd$A$ gene delivery. Most probably, this is because LV-cardiomyocytes in 6-8-day-old piglets undergo intensive physiological hypertrophy that is accompanied by a relatively high ventricular anf expression (M. Torrado and A.T. Mikhailov, unpublished data). 
Such a hypertrophic state of targeting LV-cardiomyocytes may mask or even impose a pro-hypertrophic effect of forced myocd expression in vivo. Alternately, the myocd-mediated hypertrophic effect, which has been identified in an in vitro model system, may be difficult to mimic in in vivo settings because the response of targeting LV-cardiomyocytes to elevated myocdexpression in vivo could be modified by their interactions with adjacent non-transfected working myocytes as well as with other cell types (i.e., impulse-conducting cardiomyocytes, endothelial cells, and cardiac fibroblasts) compounding neonatal LV-tissue.

In summary, our data revealed that under apparently normal hypertrophic growth the outcome of the response of the LV, as a heterotypic cell system, to elevated myocd-A expression might be maladaptive. More broadly, this observation is consistent with the suggestion (Pipes et al., 2006) that myocdoverexpression might represent a maladaptive response to pathological remodeling of the heart.

\section{Concluding remarks}

This study is the first to show possible functional consequences of forced MYOCD-A production in the LVFW resulting from direct intra-myocardial gene transfer in vivo. Despite the efficiency of our gene delivery technique, it is unrealistic to expect a $100 \%$ myocd$A$-transfection of every cell in LVFW. Nevertheless, ventricular performance was affected in experimental piglets although, on average, no more than $40 \%$ of the LVFW was efficiently transfected by myocd- $A$. The results show that even local changes in MYOCD dosage (and, consequently, in MYOCD-A/MYOCD-B ratio) may modulate the responsiveness of LV-myocardium to overload during early postnatal development. Observed transient deterioration in ventricular performance of the neonatal heart in response to a local $m y o c d-A$ overexpression in the LV myocardium suggests that cessation of ventricular myocd- $A$ expression at early-stage cardiomyopathy might represent a safety feature from a therapeutic point of view. Although this is an encouraging idea, it remains to be determined whether $m y o c d-A$ forced expression in the LV may be a maladaptive background upon stress, resulting in pathological LV remodeling. Our preliminary results indicate that in neonatal piglets LV myocd- $A$ transfection followed by adriamycininduced cardiomyopathy resulted in a significantly lower survival rate of the transfected animals compared to controls.

\section{Materials and Methods}

\section{Gene cloning and in vitro expression}

The full-length porcine myocd-A (GenBank accession no. NM_213745) and $m$ yocd- $B$ (this work, see Fig. $2 A$ ) were amplified from piglet $L V$ oligodT primed CDNA, cloned into T7 promoter-containing vector pcDNA3.1/ myc-His B (Invitrogen), verified by sequencing and expressed in vitrousing TnT T7 Quick Coupled in vitro Transcription/Translation (IVTT) System (Promega) in the presence or absence of $\left[{ }^{35} \mathrm{~S}\right]$-labeled methionine as described (Torrado et al., 2005a). The corresponding protein products were purified on Sephadex G-50 columns (Amersham Bioscience) and used in downstream SDS-PAGE and Western-blot experiments.

\section{Plasmid DNA}

The following expression plasmids in p3XFLAG-CMV-14 vector (Sigma) driven by a human cytomegalovirus (CMV) promoter/enhancer were used: (1) $m y o c d-A$ plasmid, encoding a FLAG-tagged full-length porcine myocd$A$ gene; (2) hop plasmid, encoding a FLAG-tagged full-length porcine hop
(GenBank accession no. NM_213792), (3) reporter plasmid encoding the bacterial gene for $\beta$-galactosidase ( $\beta$-ga), and (4) vector plasmid only (control empty vector). All the DNA constructs were verified by sequencing. Plasmids grown in XL1-Blue Supercompetent E. coli cells (Stratagene) were purified by using a PureLink HiPure plasmid filter purification kit (Invitrogen) according to the manufacturer's protocol. Plasmids were formulated at a final DNA concentration of $1 \mathrm{mg} / \mathrm{ml}$ in sterile isotonic saline (PBS).

\section{Animals and experimental procedures}

Animal care and handling conformed to the guidelines of the European Commission Directive 86/609/EEC on the protection of animals used for experimental and other scientific purposes and were approved by the Institutional Ethics Committee of the University of La Coruña. Litters of Large White domestic pigs were maintained in the Nütinger automatic nursery system, randomized in five groups and assigned to receive intramyocardial injections of: (1) myocd-A plasmid DNA ( $\mathrm{n}=16)$, (2) hopplasmid DNA ( $n=6)$, (3) $\beta$-gal-plasmid DNA $(n=6)$, (4) vector alone plasmid $(n=8)$, and (5) $2 \%$ Indian ink (Winsor \& Newton, UK) suspension in PBS $(n=6)$. Neonatal 6-day-old piglets were sedated, intubated, and anesthetized. While under anesthesia and mechanical ventilatory support, ECG was monitored followed by trans-costotranversal inserting a fiber-optic catheter (Cardio-Optics Inc.) and endoscopic tubular ( $3 \mathrm{~mm}$ ) cannula into left chest cavity. The pericardium was opened, and the catheter and cannula were moved to access and visualize the ventral surface of the left ventricle free wall (LVFW). Then, the endoscopic needle was introduced into the cannula, and intramyocardial injections were performed (as detailed in the body text) in the ventro-lateral area of the LVFW. To minimize leakage of injectate from the puncture site, each injection was performed slowly under direct visualization. Intra-operative monitoring of cardiovascular parameters (blood pressure, pulse rate, body temperature) was performed during the procedure. On the $2^{\text {nd }}$ and $7^{\text {th }}$ day after gene transfer, ECG was monitored in closed-chest piglets followed by measurements of ventricular end-systolic (LV/RV-ESP) and end-diastolic pressure (LV/RVEDP) in open-chest piglets using a Dräger UM3.1 pressure transducer and a recording device (Drägerwerk AG, Germany) as described (Torrado etal., 2006). Then animals were euthanized and the hearts were rapidly excised, weighed, and photographed. All these procedures were conducted by two investigators blinded to the experimental design. The ventral LVFW of each heart was sectioned into 9 regions ( 1x1 cm each; see Fig. 3 ) which were then assayed individually for RNA and protein isolation, and tissue fixation (Torrado et al., 2004; Torrado et al., 2005b; Torrado et al., 2006).

\section{Semiquantitative $R T$-PCR}

Total RNA was extracted by RNAeasy-Mini Kit (Qiagen) according to the manufacturer's protocol, subjected to on column digestion of DNA with RNase-free DNase (Qiagen), and reverse transcribed using the SuperScript III (Invitrogen) and oligo-dT primers. Semiquantitative RT-PCR was performed as described previously (Torrado et al., 2003; Torrado et al., 2004). All PCR reactions included primers for the candidate (myocd- $A$ or $m y o c d-$ $B$ ) and control (ribosomal protein L19 - RPL19) genes. Porcine myocd- $A$ and myocd- $B$ were amplified with the oligonucleotides 273 and 270 generating 294-bp myocd-A and 438-bp myocd-BPCR-products (see Fig. $2 \mathrm{~A}, \mathrm{~B})$. Discriminating primer set for the specific amplification of delivered FLAG-tagged $m y o c d-A$ was 279 and 239 (derived from the FLAG vector sequence) for a 577-bp PCR product. The porcine RPL 19 (accession no: $\mathrm{BI} 181894$ ) was used to design oligonucleotides 36 and 65 for a 480 -bp PCR product. Reactions, including NRT (non-RT control) and NTC (no template control), were performed at least in triplicate. PCR products were visualized on $2 \%$ agarose gels by ethidium bromide $(\mathrm{EtBr})$ staining and band intensity was estimated by densitometry (VersaDoc 1000) and Quantity One software (Bio-Rad).

\section{Quantitative real-time RT-PCR (qRT-PCR)}

qRT-PCR was performed using SYBR Green Master Mix (Bio-Rad) on 
Bio-Rad IQ5 instrument as described (Torrado et al., 2006) with the addition of primer pairs targeting transcripts encoding (see Table 3 ) the porcine m/c3f(primers 292 and 293), anf(primers 266 and 267), cnn1 (primers 295 and 296), tag/n/SM-22 (primers 297 and 298), and actg2 (primers 301 and 302). The primer pairs were located in different exons to rule out genomic DNA amplification. Each primer pair used yielded a single peak of dissociation on the melting curve and a single band with the expected size on agarose gel. Identity of the PCR products was confirmed by sequencing. Non-template and non-RT RNA template reactions were used as negative controls. Results were normalized against RPL 19 expression (oligonucleotides 64 and 206). The efficiency of target and reference amplification was tested to be approximately equal. Primer sequences and additional details on RT-PCR/qRT-PCR are available upon request.

\section{$\beta$-gal enzyme activity assay}

$\beta$-gal enzyme activity was measured in LVFW extracts using a high sensitivity $\beta$-galactosidase Assay Kit (Stratagene) with standards supplied by the manufacturer at $\mathrm{pH} 8.0$ to minimize non-specific staining. Enzyme activity values were normalized to total protein concentration determined using the Bio-Rad DCProtein Assay.

\section{Antibody production}

The C-terminal part (amino acid residues 731-873; CT-MYOCD) of porcine MYOCD-A (GenBank accession no: NP_998910) was cloned into pCAL-n-FLAG vector (Stratagene) and expressed in Rosetta-gami B(DE3) (Novagen). Recombinant CT-MYOCD-A (fused to the 5-kDa tag, which included the calmodulin-binding peptide (CBP) and FLAG peptide) was purified from bacterial lysates using calmodulin-affinity resin (Stratagene) and used as immunogen. Polyclonal antibodies against porcine CTMYOCD-A were raised in rabbits by Davids Biotechnologie (Regenburg, Germany). Obtained anti-CT-MYOCD-A antibodies (marked by us as AB531) were shown to be specific for recombinant porcine MYOCD-A and MYOCD-B by Western blot with competing peptide. Five fragments covering the major part of the sequence of porcine myocd- $A$ were cloned and expressed as just described, and resulting purified products were probed by Western blot with anti-FLAG (Sigma) and our lab-derived AB531 antibodies (Fig. 4B,C). Anti-FLAG antibodies detected each of the expressed $m y 0 c d-A$ fragments, whereas $A B 531$ antibodies recognized only the CT-MYOCD-A (i.e., the immunogen); no cross-reactivity was observed with other MYOCD-A-derived fragments. These results indicated that AB531 antibodies detect only the TAD-containing CT-fragment, a region that is similar for all the myocd isoforms identified to date in cardiac and SMC-containing tissues (see Fig. 4A).

\section{SDS-PAGE and Western blotting}

LVFW tissue samples were homogenized and solubilized in standard $2 x$ Laemmli sample buffer as previously described (Torrado et al., 2006). Extracted proteins were subjected to SDS-PAGE (Mini-Protean-III, BioRad), stained with Coomassie or blotted onto PVDF membranes (Hybond$\mathrm{P}$, Amersham Bioscience), and probed with rabbit polyclonal antibodies against porcine MYOCD or mouse monoclonal anti-my $c$ or anti-FLAG antibodies (Sigma). Molecular weight (MW) standards (MARK-12 and SeeBlue Plus2 from Invitrogen, and high and low MW calibration kits from Amersham Biosciences) were included on each gel. Equivalence of protein loading was confirmed by Amido-Black staining of blots before (blotreplicas) and after immunodetection. Blocking, washing, incubation with diluted primary and secondary HRP-conjugated antibodies (Sigma), and visualization of immunodecorated bands by the Super-Signal West Pico chemiluminescent substrate (Pierce Biotechnology) was carried out as previously described (Torrado et al., 2005a; Torrado et al., 2006). IVTTsynthesized myc-tagged pig MYOCD-A or MYOCD-B was included as a positive control for anti-CT-MYOCD-A antibodies. Substitution of the primary antibodies with anti-CT-MYOCD-A antibodies neutralized by the competing peptide was included in negative controls.

\section{Histology}

LVFW tissue samples were fixed in $4 \%$ formalin in PBS and embedded in paraplast (Sigma). Serial transverse $5 \mu \mathrm{m}$-sections were subjected to haematoxylin-eosin, Masson's trichrome and von Kossa staining (Bancroft and Stevens, 1996) and examined under a "Nikon Eclipse 600" microscope by an experienced technician blinded to the gene-delivery experiments.

\section{Statistics}

Results are expressed as mean \pm SEM. Statistical significance was evaluated by Student's $t$ test. Statistical analyses were performed with the SPSS 13 software. A value of $p<0.05$ was considered statistically significant.

\section{Acknowledgements}

We thank Professor Juan Aréchaga for the invitation to contribute an article for this issue. This work was supported by a Grant (SAF200401462) from Spanish Ministry of Education and Science and by a Grant (PGIDITO4BTF16001 PR5) from the Autonomic Government of Galicia.

\section{References}

BADORFF, C., SEEGER, F.H., ZEIHER, A.M. and DIMMELER, S. (2005). Glyco gen synthase kinase 3beta inhibits myocardin-dependent transcription and hypertrophy induction through site-specific phosphorylation. Circ Res 97: 645654.

BANCROFT, J.D. and STEVENS, A. (1996). Theory and practice of histological techniques. Churchill Livingstone, Edinburgh.

CALLIS, T.E., CAO, D. and WANG, D.Z. (2005). Bone morphogenetic protein signaling modulates myocardin transactivation of cardiac genes. Circ Res 97: 992-1000.

CHEN, F., KOOK, H., MILEWSKI, R., GITLER, A.D., LU, M.M., LI, J., NAZARIAN R., SCHNEPP, R., JEN, K., BIBEN, C., RUNKE, G., MACKAY, J.P., NOVOTNY J., SCHWARTZ, R.J., HARVEY, R.P., MULLINS, M.C. and EPSTEIN, J.A (2002a). Hop is an unusual homeobox gene that modulates cardiac development. Ce//110: 713-723.

CHEN, J., KITCHEN, C.M., STREB, J.W. and MIANO, J.M. (2002b). Myocardin: a component of a molecular switch for smooth muscle differentiation. J Mol Cell Cardio/34: 1345-1356.

CHOW, N., BELL, R.D., DEANE, R., STREB, J.W., CHEN, J., BROOKS, A., VAN NOSTRAND, W., MIANO, J.M. and ZLOKOVIC, B.V. (2007). Serum response factor and myocardin mediate arterial hypercontractility and cerebral blood flow dysregulation in Alzheimer's phenotype. Proc Nat/Acad Sci USA 104: 823-828.

CREEMERS, E.E., SUTHERLAND, L.B., MCANALLY, J., RICHARDSON, J.A. and OLSON, E.N. (2006a). Myocardin is a direct transcriptional target of Mef2, Tead and Foxo proteins during cardiovascular development. Development 133: 4245-4256.

CREEMERS, E.E., SUTHERLAND, L.B., OH, J., BARBOSA, A.C. and OLSON, E.N. (2006b). Coactivation of MEF2 by the SAP domain proteins myocardin and MASTR. Mol Cel/23: 83-96.

DU, K.L., IP, H.S., LI, J., CHEN, M., DANDRE, F., YU, W., LU, M.M., OWENS, G.K. and PARMACEK, M.S. (2003). Myocardin is a critical serum response factor cofactor in the transcriptional program regulating smooth muscle cell differentiation. Mol Cel/ Bio/23: 2425-2437.

ELORANTA, J.J. and HURST, H.C. (2002). Transcription factor AP-2 interacts with the SUMO-conjugating enzyme UBC9 and is sumolated in vivo. $J$ Biol Chem 277: 30798-30804.

HENDRIX, J.A., WAMHOFF, B.R., MCDONALD, O.G., SINHA, S., YOSHIDA, T. and OWENS, G.K. (2005). 5' CArG degeneracy in smooth muscle alpha-actin is required for injury-induced gene suppression in vivo. J Clin Invest 115: 418427.

KELLY, R., ALONSO, S., TAJBAKHSH, S., COSSU, G. and BUCKINGHAM, M. (1995). Myosin light chain $3 F$ regulatory sequences confer regionalized cardiac and skeletal muscle expression in transgenic mice. J Cel/ Bio/129: 383-396.

KELLY, R.G., ZAMMIT, P.S., SCHNEIDER, A., ALONSO, S., BIBEN, C. and BUCKINGHAM, M.E. (1997). Embryonic and fetal myogenic programs act 
through separate enhancers at the MLC1F/3F locus. Dev Bio/187: 183-199.

KOOK, H. and EPSTEIN, J.A. (2003). Hopping to the beat. Hop regulation of cardiac gene expression. Trends Cardiovasc Med 13: 261-264.

LI, H.J., HAQUE, Z., LU, Q., LI, L., KARAS, R. and MENDELSOHN, M. (2007). Steroid receptor coactivator 3 is a coactivator for myocardin, the regulator of smooth muscle transcription and differentiation. Proc Natl Acad Sci USA 104: 4065-4070.

LI, S., WANG, D.Z., WANG, Z., RICHARDSON, J.A. and OLSON, E.N. (2003). The serum response factor coactivator myocardin is required for vascular smooth muscle development. Proc Nat/ Acad Sci USA 100: 9366-9370.

LIU, N. and OLSON, E.N. (2006). Coactivator control of cardiovascular growth and remodeling. Curr Opin Cell Biol18: 715-722.

LONG, X., CREEMERS, E.E., WANG, D.Z., OLSON, E.N. and MIANO, J.M. (2007). Myocardin is a bifunctional switch for smooth versus skeletal muscle differentiation. Proc Natl Acad Sci USA 104: 16570-16575.

MCGREW, M.J., BOGDANOVA, N., HASEGAWA, K., HUGHES, S.H., KITSIS, R.N. and ROSENTHAL, N. (1996). Distinct gene expression patterns in skeletal and cardiac muscle are dependent on common regulatory sequences in the MLC1/3 locus. Mol Cel/ Bio/16: 4524-4534.

MICHAEL, A., HAQ, S., CHEN, X., HSICH, E., CUI, L., WALTERS, B., SHAO, Z., BHATTACHARYA, K., KILTER, H., HUGGINS, G., ANDREUCCI, M., PERIASAMY, M., SOLOMON, R.N., LIAO, R., PATTEN, R., MOLKENTIN, J.D. and FORCE, T. (2004). Glycogen synthase kinase-3beta regulates growth, calcium homeostasis, and diastolic function in the heart. $J$ Biol Chem 279: 21383-21393.

MULLER-BORER, B.J., CASCIO, W.E., ESCH, G.L., KIM, H.S., COLEMAN, W.B., GRISHAM, J.W., ANDERSON, P.A. and MALOUF, N.N. (2007). Mechanisms controlling the acquisition of a cardiac phenotype by liver stem cells. Proc Nat/ Acad Sci USA 104: 3877-3882.

OH, J., WANG, Z., WANG, D.Z., LIEN, C.L., XING, W. and OLSON, E.N. (2004). Target gene-specific modulation of myocardin activity by GATA transcription factors. Mol Cell Bio/24: 8519-8528.

OKA, T., XU, J. and MOLKENTIN, J.D. (2007). Re-employment of developmental transcription factors in adult heart disease. Semin Cell Dev Biol18: 117-131.

OLSON, E.N. (2004). A decade of discoveries in cardiac biology. Nat Med10: 467474.

PARLAKIAN, A., CHARVET, C., ESCOUBET, B., MERICSKAY, M., MOLKENTIN, J.D., GARY-BOBO, G., DE WINDT, L.J., LUDOSKY, M.A., PAULIN, D., DAEGELEN, D., TUIL, D. and LI, Z. (2005). Temporally controlled onset of dilated cardiomyopathy through disruption of the SRF gene in adult heart. Circulation 112: 2930-2939.

PARMACEK, M.S. (2007). Myocardin-related transcription factors: critical coactivators regulating cardiovascular development and adaptation. Circ Res 100: 633-644.

PIPES, G.C., CREEMERS, E.E. and OLSON, E.N. (2006). The myocardin family of transcriptional coactivators: versatile regulators of cell growth, migration, and myogenesis. Genes Dev20: 1545-1556.

PIPES, G.C., SINHA, S., QI, X., ZHU, C.H., GALLARDO, T.D., SHELTON, J., CREEMERS, E.E., SUTHERLAND, L., RICHARDSON, J.A., GARRY, D.J., WRIGHT, W.E., OWENS, G.K. and OLSON, E.N. (2005). Stem cells and their derivatives can bypass the requirement of myocardin for smooth muscle gene expression. Dev Bio/288: 502-513.

QIU, P., RITCHIE, R.P., FU, Z., CAO, D., CUMMING, J., MIANO, J.M., WANG, D.Z., LI, H.J. and LI, L. (2005). Myocardin enhances Smad3-mediated transforming growth factor-beta1 signaling in a CArG box-independent manner: Smadbinding element is an important cis element for SM22alpha transcription in vivo. Circ Res 97: 983-991.

SAPETSCHNIG, A., RISCHITOR, G., BRAUN, H., DOLL, A., SCHERGAUT, M., MELCHIOR, F. and SUSKE, G. (2002). Transcription factor Sp3 is silenced through SUMO modification by PIAS1. EMBO J21: 5206-5215.

SHIN, C.H., LIU, Z.P., PASSIER, R., ZHANG, C.L., WANG, D.Z., HARRIS, T.M., YAMAGISHI, H., RICHARDSON, J.A., CHILDS, G. and OLSON, E.N. (2002). Modulation of cardiac growth and development by HOP, an unusual homeodomain protein. Cel/110: 725-735.

SMALL, E.M., WARKMAN, A.S., WANG, D.Z., SUTHERLAND, L.B., OLSON, E.N. and KRIEG, P.A. (2005). Myocardin is sufficient and necessary for cardiac gene expression in Xenopus. Development 132: 987-997.

TORRADO, M., LOPEZ, E., CENTENO, A., CASTRO-BEIRAS, A. and MIKHAILOV, A.T. (2004). Left-right asymmetric ventricular expression of CARP in the piglet heart: regional response to experimental heart failure. Eur J Heart Fail6: 161 172.

TORRADO, M., LOPEZ, E., CENTENO, A., MEDRANO, C., CASTRO-BEIRAS, A. and MIKHAILOV, A.T. (2003). Myocardin mRNA is augmented in the failing myocardium: expression profiling in the porcine model and human dilated cardiomyopathy. J Mol Med81: 566-577.

TORRADO, M., NESPEREIRA, B., BOUZAMAYOR, Y., CENTENO, A., LOPEZ, E. and MIKHAILOV, A.T. (2006). Differential atrial versus ventricular ANKRD1 gene expression is oppositely regulated at diastolic heart failure. FEBS Lett 580: $4182-4187$

TORRADO, M., NESPEREIRA, B. and MIKHAILOV, A.T. (2005a). Fetal cardiac control genes: implications for postnatal heart growth and heart disease. Trends Dev Biol1: 27-38.

TORRADO, M., NESPEREIRA, B., LOPEZ, E., CENTENO, A., CASTRO-BEIRAS A. and MIKHAILOV, A.T. (2005b). ANKRD1 specifically binds CASQ2 in heart extracts and both proteins are co-enriched in piglet cardiac Purkinje cells. JMO/ Cell Cardio/38: 353-365.

VAN TUYN, J., ATSMA, D.E., WINTER, E.M., VAN DER VELDE-VAN DIJKE, I., PIJNAPPELS, D.A., BAX, N.A., KNAAN-SHANZER, S., GITTENBERGER-DE GROOT, A.C., POELMANN, R.E., VAN DER LAARSE, A., VAN DER WALL, E.E., SCHALIJ, M.J. and DE VRIES, A.A. (2007a). Epicardial cells of human adults can undergo an epithelial-to-mesenchymal transition and obtain characteristics of smooth muscle cells in vitro. Stem Cel/s 25: 271-278.

VAN TUYN, J., PIJNAPPELS, D.A., DE VRIES, A.A.F., DE VRIES, I., VAN DER VELDE-VAN DIJKE, I., KNAAN-SHANZER, S., VANDER LAARSE, A., SCHALIJ, M.J. and ATSMA, D.E. (2007b). Fibroblasts from human postmyocardial infarction scars acquire properties of cardiomyocytes after transduction with a recombinant myocardin gene. FASEB J21: 3369-3379.

VAN TUYN, J., KNAAN-SHANZER, S., VAN DE WATERING, M.J., DE GRAAF, M., VAN DER LAARSE, A., SCHALIJ, M.J., VAN DER WALL, E.E., DE VRIES, A.A. and ATSMA, D.E. (2005). Activation of cardiac and smooth muscle-specific genes in primary human cells after forced expression of human myocardin. Cardiovasc Res 67: 245-255.

WANG, D., CHANG, P.S., WANG, Z., SUTHERLAND, L., RICHARDSON, J.A. SMALL, E., KRIEG, P.A. and OLSON, E.N. (2001). Activation of cardiac gene expression by myocardin, a transcriptional cofactor for serum response factor. Cel/105: 851-862.

WANG, D.Z., LI, S., HOCKEMEYER, D., SUTHERLAND, L., WANG, Z., SCHRATT, G., RICHARDSON, J.A., NORDHEIM, A. and OLSON, E.N. (2002). Potentiation of serum response factor activity by a family of myocardin-related transcription factors. Proc Nat/ Acad Sci USA 99: 14855-14860.

WANG, J., LI, A., WANG, Z., FENG, X., OLSON, E.N. and SCHWARTZ, R.J. (2007). Myocardin sumoylation transactivates cardiogenic genes in pluripotent 10T1/2 fibroblasts. Mol Cel/ Bio/27: 622-632.

WANG, Z., WANG, D.Z., HOCKEMEYER, D., MCANALLY, J., NORDHEIM, A. and OLSON, E.N. (2004). Myocardin and ternary complex factors compete for SRF to control smooth muscle gene expression. Nature 428:185-189.

WANG, Z., WANG, D.Z., PIPES, G.C. and OLSON, E.N. (2003). Myocardin is a master regulator of smooth muscle gene expression. Proc Nat/ Acad Sci USA 100: 7129-7134

XING, W., ZHANG, T.C., CAO, D., WANG, Z., ANTOS, C.L., LI, S., WANG, Y., OLSON, E.N. and WANG, D.Z. (2006). Myocardin induces cardiomyocyte hypertrophy. Circ Res 98: 1089-1097.

YOSHIDA, T., KAWAI-KOWASE, K. and OWENS, G.K. (2004). Forced expression of myocardin is not sufficient for induction of smooth muscle differentiation in multipotential embryonic cells. Arterioscler Thromb Vasc Bio/24: 1596-1601.

YOSHIDA, T., SINHA, S., DANDRE, F., WAMHOFF, B.R., HOOFNAGLE, M.H., KREMER, B.E., WANG, D.Z., OLSON, E.N. and OWENS, G.K. (2003). Myocardin is a key regulator of carg-dependent transcription of multiple smooth muscle marker genes. Circ Res 92: 856-865.

ZHANG, X., AZHAR, G., ZHONG, Y. and WEI, J.Y. (2004). Identification of a novel serum response factor cofactor in cardiac gene regulation. J Biol Chem 279: 55626-55632. 


\section{Related, previously published Int. J. Dev. Biol. articles}

See our recent Special Issue Ear Development edited by Fernando Giraldez and Bernd Fritzsch at: http://www.ijdb.ehu.es/web/contents.php?vol=51\&issue=6-7

See our Special Issue Mammalian Reproduction \& Development in honor of Anne McLaren and edited by Brigid Hogan at: http:// www.ijdb.ehu.es/web/contents.php?vol=45\&issue=3

Myoskeletin, a factor related to Myocardin, is expressed in somites and required for hypaxial muscle formation in Xenopus

Hui Zhao, Martha L. Rebbert and Igor B. Dawid

Int. J. Dev. Biol. (2007) 51: 315-320

Heart formation and left-right asymmetry in separated right and left embryos of a newt

Kazuhiro Takano, Yuzuru Ito, Shuichi Obata, Tsutomu Oinuma, Shinji Komazaki, Hiroaki Nakamura and Makoto Asashima

Int. J. Dev. Biol. (2007) 51: 265-272

Thyroid hormone receptor expression in the obligatory paedomorphic salamander Necturus maculosus

Virginie Vlaeminck-Guillem, Rachid Safi, Philippe Guillem, Emmanuelle Leteurtre, Martine Duterque-Coquillaud and Vincent Laudet

Int. J. Dev. Biol. (2006) 50: 553-560

\section{Biophysical mechanisms of cardiac looping \\ Larry A. Taber \\ Int. J. Dev. Biol. (2006) 50: 323-332}

Biophysical regulation during cardiac development and application to tissue engineering

Sharon Gerecht-Nir, Milica Radisic, Hyoungshin Park, Christopher Cannizzaro, Jan Boublik, Robert Langer and Gordana Vunjak-Novakovic

Int. J. Dev. Biol. (2006) 50: 233-243

The cap ' $n$ ' collar family member NF-E2-related factor 3 (Nrf3) is expressed in mesodermal derivatives of the avian embryo

Heather C. Etchevers

Int. J. Dev. Biol. (2005) 49: 363-367

Amphibian in vitro heart induction: a simple and reliable model for the study of vertebrate cardiac development

Takashi Ariizumi, Masayoshi Kinoshita, Chika Yokota, Kazuhiro Takano, Keiichi

Fukuda, Nobuo Moriyama, George M Malacinski and Makoto Asashima

Int. J. Dev. Biol. (2003) 47: 405-410

Induction and patterning of the cardiac conduction system

David J Pennisi, Stacey Rentschler, Robert G Gourdie, Glenn I Fishman and Takashi Mikawa

Int. J. Dev. Biol. (2002) 46: 765-775

Efficient Cre-mediated deletion in cardiac progenitor cells conferred by a 3'UTR-ires-Cre allele of the homeobox gene Nkx2-5

Edouard G Stanley, Christine Biben, Andrew Elefanty, Louise Barnett, Frank Koentgen, Lorraine Robb and Richard P Harvey

Int. J. Dev. Biol. (2002) 46: 431-439

Heat shock factor 2 is activated during mouse heart development $M$ Eriksson, E Jokinen, L Sistonen and S Leppä Int. J. Dev. Biol. (2000) 44: 471-477

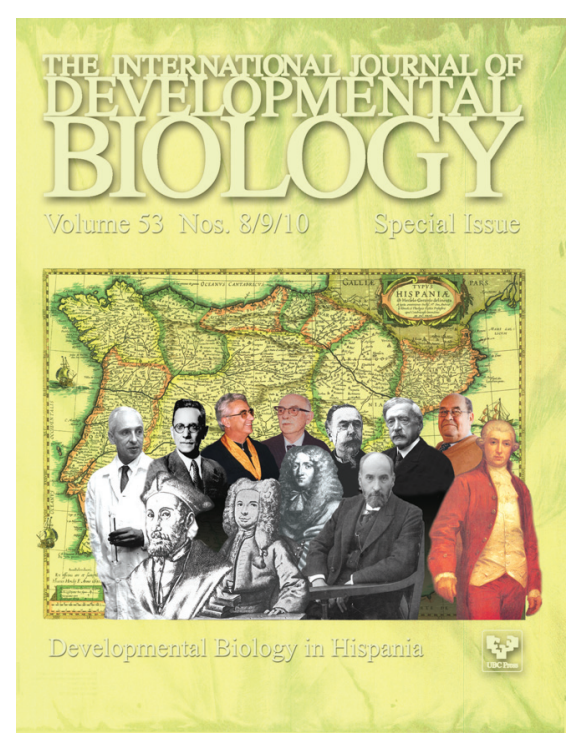

5 yr ISI Impact Factor $(2008)=3.271$

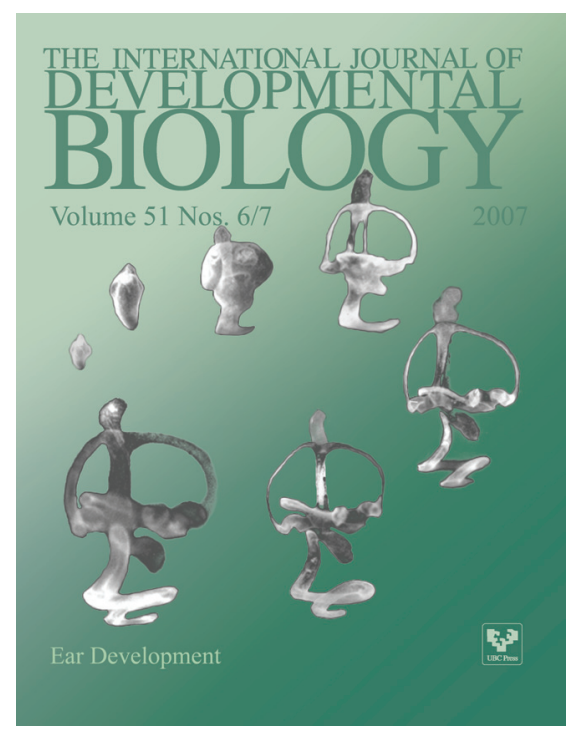

